\title{
Neural Dysfunction in Cognitive Control Circuits in Persons at Clinical High-Risk for Psychosis
}

\author{
Tiziano Colibazzi,,', Guillermo Horga', Zhishun Wang ${ }^{1,2}$, Yuankai Huo ${ }^{1,2}$, Cheryl Corcoran', Kristin Klahr ${ }^{1,2}$, \\ Gary Brucato', Ragy Girgis', Kelly Gill', Anissa Abi-Dargham' and Bradley S Peterson ${ }^{3}$ \\ 'Department of Psychiatry, The New York State Psychiatric Institute, Columbia College of Physicians and Surgeons, New York, NY, USA; ${ }^{2}$ Center for \\ Developmental Neuropsychiatry, The New York State Psychiatric Institute, Columbia College of Physicians and Surgeons, New York, NY, USA; \\ ${ }^{3}$ Institute for the Developing Mind, Children's Hospital Los Angeles, Keck School of Medicine at the University of Southern California, Los Angeles, \\ CA, USA
}

Cognitive control, a set of functions that develop throughout adolescence, is important in the pathogenesis of psychotic disorders. Whether cognitive control has a role in conferring vulnerability for the development of psychotic illness is still unknown. The aim of this study was to investigate the neural systems supporting cognitive control in individuals deemed to be potentially prodromal for psychotic illness. We recruited 56 participants at clinical high-risk (CHR) for psychosis based on the Structured Interview for Psychosis-Risk Syndromes (SIPS) and 49 healthy controls. Twelve of the CHR participants eventually developed psychosis. We compared functional magnetic resonance imaging ( $\mathrm{MMRI}$ ) BOLD signal during the performance of the Simon task. We tested for differences between $\mathrm{CHR}$ individuals and controls in conflict-related functional activity. In the CHR group when compared with controls, we detected smaller conflictrelated activations in several cortical areas, including the Dorsolateral Prefrontal Cortex (DLPFC). Furthermore, conflict-related activations in the DLPFC of those CHR individuals who ultimately developed psychosis (CHR converters) were smaller than in non-converters (CHR non-converters). Higher levels of conflict-related activation were associated with better social and role outcome. Risk for psychosis was associated at the neural level with reduced conflict-related brain activity. This neural phenotype appears correlated within the DLPFC with the development of psychosis and with functional outcome.

Neuropsychopharmacology (2015) 4I, I24I-1250; doi: I0.1038/npp.20 15.273; published online 4 November 20I5

\section{INTRODUCTION}

Adolescence is an important time for cognitive and emotional development (Crone and Dahl, 2012). Successful transition into adult life requires the development of cognitive control, which supports the coordination of a vast array of cognitive functions, including the ability to negotiate competing demands and to manage conflict between different behavioral responses to life circumstances. Many psychiatric disorders make their first appearance in adolescence (Paus et al, 2008), when these control processes become established and the demands of the environment for conforming behavior increase sharply. Of those disorders, psychotic illness is among the most functionally devastating.

Neurocognitive abnormalities, particularly in cognitive control, may be a core feature of psychotic illness and predict its functional outcome (Snitz et al, 2006; Lesh et al, 2011; Carrion et al, 2011, 2013; Wood et al, 2003; Green,

*Correspondence: Professor T Colibazzi, Division of Translational Imaging, College of Physicians and Surgeons and New York State Psychiatric Institute, Office 6104, 1051 Riverside Drive, Unit 74, New York, NY 10032, USA, Tel: + I 212561 89|I, Fax: + | 212561 8734, E-mail: tc2237@columbia.edu

Received 15 March 2015; revised 22 August 2015; accepted 23 August 2015; accepted article preview online 10 September 2015
1996). However, whether these cognitive phenomena are a cause of the illness, a consequence of it, or are an effect of disease chronicity, medication use, or other disease-related factors, is still unknown. Even less is known as to the presence and the role in the pathogenesis of psychosis of abnormalities in the functional circuits that support cognitive control.

The study of the neural circuits mediating cognitive control during the phase preceding the onset of psychotic illness is of particular interest in disentangling these various possibilities, given the general absence of these confounding variables at that time. This phase of illness, called clinical high-risk (CHR) or psychosis prodrome, is characterized by attenuated psychotic symptoms, brief psychotic symptoms, or functional decline in the presence of genetic risk (Yung and McGorry, 1996). A percentage of CHR individuals, identified based on standard clinical criteria (Miller et al, 2003), develop psychosis within 2 years of identification (Wood et al, 2003; Cannon et al, 2008). Recent reports suggest that most of these individuals continue to be chronically impaired, whether or not they eventually develop the full psychotic syndrome (Carrion et al, 2013).

We aimed to determine the nature and extent of abnormalities in the neural systems that support cognitive control in CHR adolescents and young adults. We used the 
Simon task to test the hypothesis that CHR individuals have impaired functioning of the frontostriatal systems responsible for processing cognitive conflict. In this paradigm, which probes cognitive control and self-regulation more generally, conflict is generated when the task-required, less automatic response to the direction of an arrow, conflicts with the more automatic and prepotent response to the side of the screen on which the arrow appears (incongruent trial) (Kerns et al, 2004; Botvinick et al, 1999; Egner, 2007, 2008). This task allows for investigation of the neural processes that support the processing and resolution of cognitive conflict (Egner, 2007).

Although functional abnormalities have been observed previously in both the cortex and the striatum of CHR individuals, the exact nature of these abnormalities requires further explication. Based on prior studies indicating the presence of abnormal neural processes when performing tasks that place demands on cognitive control (Niendam et al, 2013), and given extensive clinical and developmental considerations that suggest the presence of impaired capacities for processing and resolving conflict in psychotic illness (Luna, 2009), we hypothesized that CHR individuals would present with a neural impairment of conflict processing, an aspect of cognitive control. Mastering the ability to regulate cognitive control flexibly based on changing demands is especially crucial for establishing adaptive, goal-directed behaviors and, thus, for a successful transition from adolescence into adulthood.

Our primary hypothesis was that CHR individuals would exhibit, when compared with healthy controls, reduced neural activity in DLPFC and dorsal striatum during the processing of conflict (Niendam et al, 2013).

We focused our analyses on post-congruent conflict (ie, differential neural activity when processing incongruent, conflict-laden stimuli compared with processing congruent, conflict-free stimuli, when both stimulus types immediately follow congruent stimuli). This priori choice is motivated by theoretical, statistical, and empirical reasons. First postcongruent conflict produces the greatest conflict in both neural and behavioral measures (Marsh et al, 2011, 2013), thus allowing us to detect group differences with greater sensitivity. Second, a vast literature describing cognitive interference has established the presence of sizeable sequential effects known as the 'conflict adaptation' or 'Gratton' effect (Egner, 2008; Horga et al, 2011), wherein both behavioral and BOLD data are best explained as an interaction between current and preceding congruence. This established interaction thus complicates the interpretation of main effects of the so-called 'standard' Simon or currentcongruence effect (I vs C) without regard for the effects of the preceding trial on task performance. We thus focused $a$ priori on post-congruent conflict for these reasons and following our prior work (Marsh et al, 2011, 2013).

We expected that this decrease in conflict-related functional activation would be related to the severity of positive symptoms, conversion to psychosis, and to functional outcome. Our hypothesis that such abnormalities would be represented in conflict-related activity of the DLPFC and striatum was further grounded in (1) increasing evidence of abnormal functioning of frontostriatal loops in the prodromal phase of psychotic illness (Allen et al, 2012; Dandash et al, 2013; Fornito et al, 2013; Fusar-Poli et al, 2011, 2010);
(2) prior studies implicating frontostriatal circuits in cognitive control (Marsh et al, 2009; Kerns, 2006; Rubia et al, 2006); and (3) studies pointing to the selective vulnerability of the dopaminergic system in these circuits during adolescent development (Naneix et al, 2013; Matthews et al, 2013).

\section{MATERIALS AND METHODS}

\section{Participants}

Our sample consisted of 58 CHR individuals enrolled in the COPE (Center of Prevention and Evaluation) clinic at the New York State Psychiatric Institute and 55 age- and gendermatched healthy controls with mean ages of 20.79 (SD 4.00) and 21.15 (SD 3.73), respectively. CHR participants and controls were also matched for minority status (here defined as being non-Caucasian). We excluded 8 subjects who performed poorly on the task based on the behavioral exclusion criterion, leading to a final sample consisting of 56 CHR individuals and 49 healthy controls still matched for age, gender, and minority status (Table 1). The results of our analyses were not meaningfully affected when we excluded these participants. Risk for psychosis was assessed using the Structured Interview for Psychosis-Risk Syndromes (SIPS) (Miller et al, 2003). Individuals were deemed to be at CHR for psychosis if they met any of the following criteria: (1) attenuated psychotic symptoms; (2) intermittent psychotic symptoms; or (3) concurrent genetic risk and functional deterioration. CHR individuals were followed up for 2.5 years with a mean time to conversion of 18.25 months (SD 12.6). Twelve participants in this CHR sample eventually developed psychosis (20.7\% of our sample). One participant was not retained in these analyses based on our behavioral exclusion criterion leaving a total of 11 converters for our analyses. Ten CHR individuals were on antipsychotic medication at the time of scanning (including 3 out of the 10 individuals who went on to develop psychosis). Functional outcome was measured using the Global Functioning Scale: Social (GSF:S) and the Global Functioning Scale: Role (GSF:R) (Cornblatt et al, 2007) with scores equal to 6 or below indicating poor outcome and scores equal to 7 or higher indicating good outcome (Carrion et al, 2013). At last follow-up (mean time to last follow-up $=21.06$ months; $\mathrm{SD}=15.69$ ), available scores for our CHR cohort pointed to a moderate level of impairment in role functioning (mean = $6.59 ; n=44 ; \mathrm{SD}=1.37)$ and a serious level of impairment in social and interpersonal functioning (mean $=5.93 ; n=45$; $\mathrm{SD}=2.85$ ) (refer to Table 1 for the final sample retained for our analyses). The percentages of CHR individuals in our cohort who had scores equal or lower than 6 on the GFS:S and GSF:R were 43.2 and $44.4 \%$, respectively. These social and role outcomes were similar to those of other CHR cohorts (Carrion et al, 2013) as were our rates of conversion to psychosis and exposure to antipsychotic medications (Niendam et al, 2013; Dandash et al, 2013; Yaakub et al, 2013). One CHR individual was scanned around the time of conversion to psychosis. Because this subject was not an outlier and our results did not change after it was removed from the analyses, we retained this individual (refer to Supplementary Material). Additionally, we did not want to exclude a priori one end of the prodromal arc, given the importance of capturing changes on a dimensional 
Table I Demographic and Clinical Characteristics of Study Participants

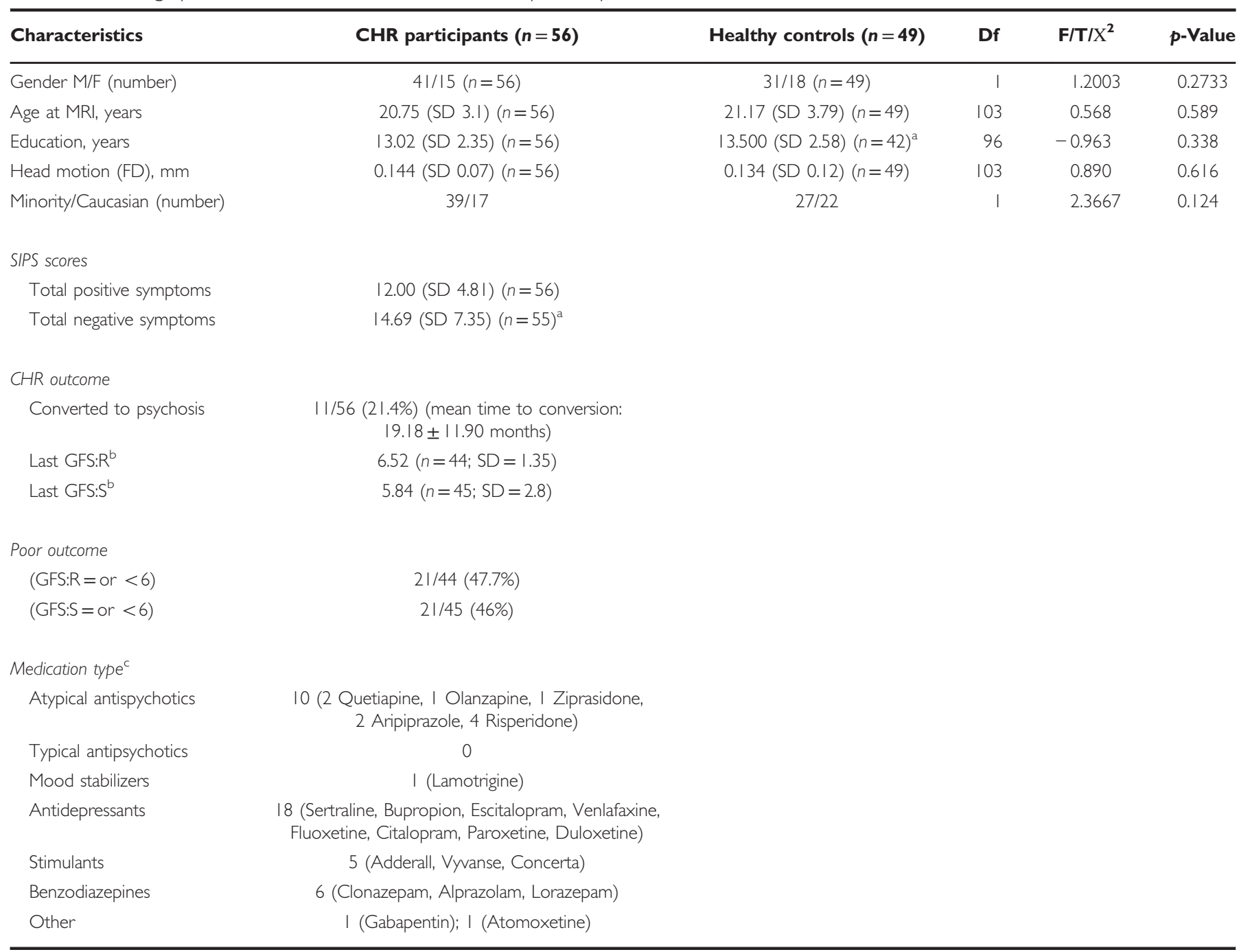

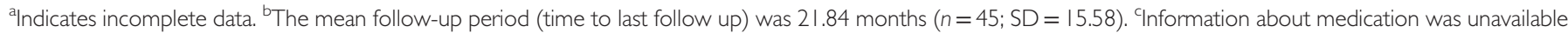
for seven participants.

continuum as subjects move toward conversion. Finally, the procedures of this study were approved by the Institutional Review Board of the New York State Psychiatric Institute.

\section{Experimental Design}

As described elsewhere (Horga et al, 2011), participants performed the Simon spatial compatibility task, a non-verbal analog of the Stroop task, during fMRI scanning. Before fMRI scanning, the task was explained to all participants, and they were required to practice five trials outside the scanner to assure their understanding of the instructions. During fMRI scanning, participants viewed the stimuli using LCD goggles (Resonance Technology, Northridge, CA) and made their responses using an MRI-compatible mouse. Stimuli were presented using E-Prime software (Psychology Software Tools, Pittsburgh, PA). Right- or left-pointing white arrows were displayed on either the left or right half of a black screen. Stimuli were congruent $(C)$ if the arrow pointed in the same direction as their position on the screen or incongruent (I) if pointing in the opposite direction as their position on the screen. Each of the arrows was displayed for $1.3 \mathrm{~s}$. Participants were instructed to press a right button if the arrow pointed right and a left button if the arrow pointed left, regardless of the position of the arrow on the screen. Inter-stimulus intervals were jittered 4-7 s. Based on prior work indicating strong sequential effects, especially firstorder effects (Egner, 2007; Marsh et al, 2013; Horga et al, 2011) during conflict processing, we divided all trials for which responses were correct into four conditions based on the combinations of the two current trial types (congruent and incongruent) and the two types of immediately preceding trials (congruent and incongruent) (Marsh et al, 2013). We designated the four conditions cC, iC, cI, or iI. Capital letters $\mathrm{C}$ and I indicate that the current trials were congruent or incongruent, and lower case letters $\mathrm{c}$ and $\mathrm{i}$ indicate that the preceding trials were congruent or incongruent, respectively. In total, the task consisted of 3 runs of 55 stimuli each, with congruent and incongruent stimuli present in equal number. Because our main contrast is post-congruent interference, ie, cI-cC, we imposed the criterion that subjects must have at least 20 correct $\mathrm{cC}$ trials 
and 20 correct cI trials. This means that we only included in the analyses subjects with a minimum number of correct trials above $60 \%$ of the total trials (which corresponds to a probability of $\sim 5 \%$ of chance performance).

Based on this exclusion criterion, we report results based on a data set comprising $56 \mathrm{CHR}$ individuals and 48 healthy controls. As shown in the accuracy plot most of the remaining subjects performed at near-ceiling level for accuracy (Supplementary Figure S1), as we had intended by using a low-demand cognitive task.

\section{Behavioral Analysis}

Mean reaction times (RTs) for each of the four trial types (cI, $\mathrm{cC}$, iI, and $\mathrm{iC}$ ) were entered into a repeated measures ANOVA with two within-subject factors (current and preceding congruence), each having two levels (congruent or incongruent), and group membership as a betweensubject factor having two levels. Interference scores were derived for each participant by subtracting the mean RT for all current congruent trials from the mean RT for all current incongruent trials. No influential outliers were found in the behavioral data. We also compared RTs for CHR converters and CHR non-converters on the trials used in the main contrast by using a two-sample $t$-test as well as its nonparametric equivalent, the Wilcoxon rank-sum test.

\section{Image Acquisition}

Imaging was performed on a 3T GE Signa whole body scanner (Milwaukee, WI) using a GE quadrature head coil. T1-weighted localizer images were acquired for positioning of the axial functional images along the anterior commissure-posterior commissure (AC-PC) line. A 3D spoiled gradient recall (SPGR) image was acquired for the coregistration of functional images with the MNI template. Parameters for acquisition of echoplanar functional images were $\mathrm{TR}=2200 \mathrm{~ms} ; \mathrm{TE}=30 \mathrm{~ms} ;$ flip angle $=90^{\circ}$; field of view $=24 \mathrm{~cm} \times 24 \mathrm{~cm}$; acquisition matrix $=64 \times 64$; slice thickness $=3.5 \mathrm{~mm}$; gap $=0 \mathrm{~mm}$; effective resolution $=$ $3.75 \mathrm{~mm} \times 3.75 \mathrm{~mm} \times 3.5 \mathrm{~mm}$; whole brain coverage; 34 slices per volume. We acquired three runs of functional data, 140 volumes per run.

\section{Preprocessing of Functional Image Data}

We used SPM8 under MATLAB 2009B to preprocess the functional imaging data (http://www.fil.ion.ucl.ac.uk/spm/). The preprocessing procedure included: (a) slice-timing correction using the middle slice of each run as the reference image; (b) motion correction for three translational directions and rotations using a rigid-body transform; (c) spatial normalization to the standard MNI template using a hybrid algorithm of affine transform and non-linear warping. Each participant's high-resolution SPGR structural image was normalized to the template, and these participant-specific warping parameters were then used to normalize the functional images to the same template; (d) resampling of the normalized functional images to $3 \times 3 \times 3 \mathrm{~mm}$ voxels; (e) Gaussian spatial filtering with an FWHM of $8 \mathrm{~mm}$. A discrete cosine transform-based high-pass filter with a basis function length of $128 \mathrm{~s}$ was used to remove low-frequency noise, such as scanner drift, from the baseline image intensity. Finally, because group differences between CHR participants and controls could yield spurious activations, we compared Frame-Wide Displacement (FD), an index of head motion, between CHR participants and healthy controls and found no significant difference between the two groups, thus ensuring that $\mathrm{CHR}$ and control groups were matched for head motion. We also applied Art Repair, an advanced motion detection and repair algorithm (https://cibsr.stan ford.edu/tools/human-brain-project/artrepair-software.html) before preprocessing.

\section{Statistical Analyses of Functional Imaging Data}

First-level analysis. Using the general linear model (GLM) as implemented in SPM8, we modeled the data for each participant with six independent functions and a constant for each run. The six independent functions corresponded to six events recorded in the task, each of which was generated by convolving a canonical hemodynamic response function (HRF) with a boxcar function derived from the onsets and durations of each of the events (cC, iC, cI and iI), fixation, and an incorrect trial term including both incorrect and missed trials. The model was estimated using the Restricted Maximum Likelihood algorithm, and then task-related contrast images were generated. (1) The contrast for our main hypothesis was [CI-cC]. We chose this contrast based on (1) several studies showing that it best identifies brain regions whose activity is associated with cognitive conflict (Marsh et al, 2013; Horga et al, 2011); (2) behavioral data from our cohort (Figure 1); (3) and previous studies suggesting that the highest difference in RTs is between cI and $\mathrm{cC}$, which can be thought behaviorally as the most and least demanding conditions, respectively (Marsh et al, 2013). This contrast captures neural activity during the current processing of conflict (I or C) in trials preceded by a congruent trial (c), thus representing conflict-related activation. We did not include incorrect trials because we wanted to exclude error-related activations and oddball-related activations (errors were infrequent in this sample). A vast literature describing cognitive interference has definitively established the presence of sizeable sequential effects known as the 'conflict adaptation' or 'Gratton' effect (Egner, 2008; Horga et al, 2011), wherein both behavioral and BOLD data are best explained as an interaction between current and preceding congruence. This established interaction thus complicates the interpretation of main effects of the so-called 'standard' Simon analysis of current congruence, or I vs C, a complication that is circumvented by focusing on postcongruent conflict.

Second-level analysis. We implemented a Bayesian posterior inference approach (Neumann and Lohmann, 2003) for the second-level analysis of the contrast images generated from the first-level GLM-based analysis. We used this Bayesian second-level approach to detect the random effects of task-related activity within and between the diagnostic groups.

Unlike classical parametric inference, which detects a group effect in a statistical parametric map by disproving the null hypothesis $(\beta=0)$ at each voxel of the image, a group effect using the Bayesian method infers the posterior 

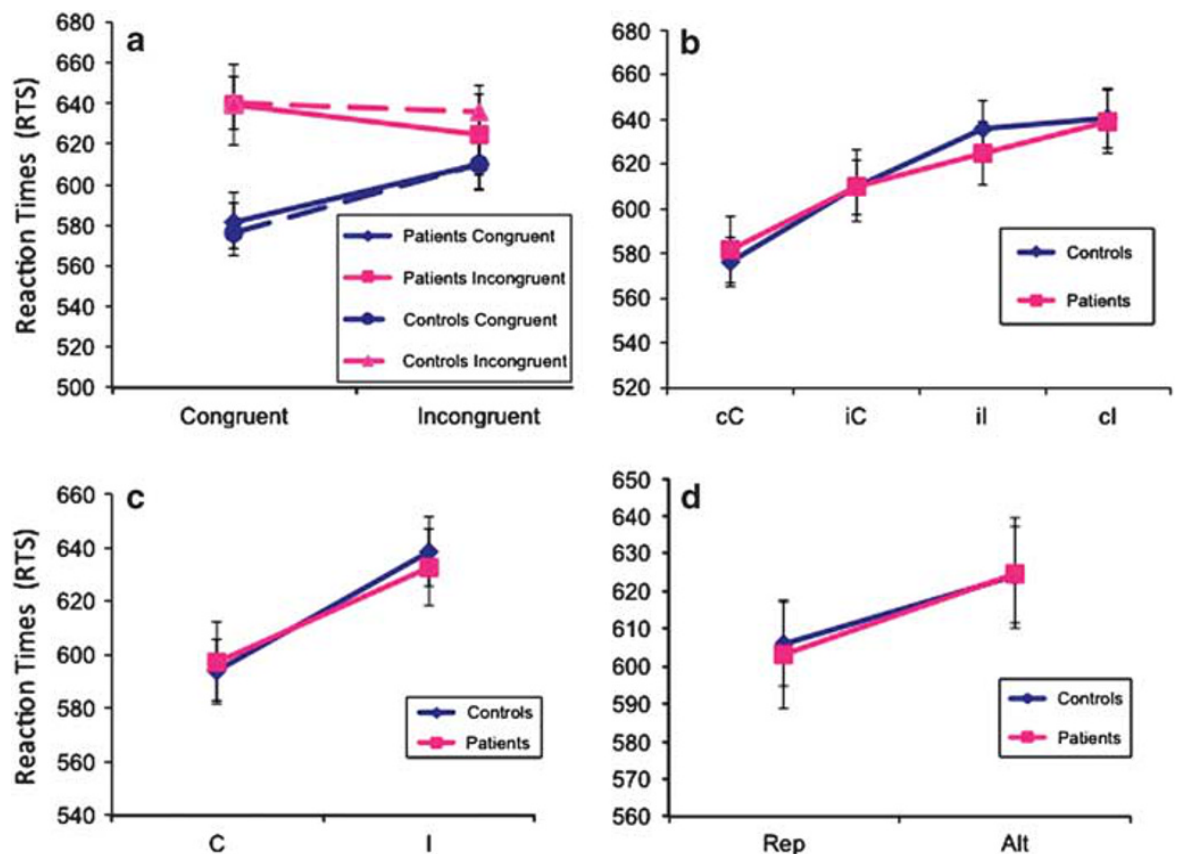

Figure I Reaction times. (a) RTs (in milliseconds) for current trials are displayed on the $Y$ axis and plotted as a function of the type of trial (c or i) preceding the current trial $(\mathrm{C}$ or I). Blue indicates current congruent trials $(C)$ and pink indicates current incongruent trials (I). The broken line refers to controls and the solid line to CHR participants. The difference in RT between incongruent and congruent trials is usually reduced if the preceding trial is also incongruent. This reduction in RT difference, known as Gratton effect or congruency sequence effect, was observed in our data. We detected no statistically significant differences in RTs between CHR participants and controls. (b) RTs in milliseconds are displayed on the $Y$ axis as a function of current trial type going from the easiest to the most difficult. cC: congruent trial preceded by congruent trial; cl: incongruent trial preceded by congruent trial; iC: congruent trial preceded by incongruent trial; il: incongruent trial preceded by incongruent trial. CHR participants and healthy controls engage equally during performance of the Simon task. Between-group differences in reaction times across the same condition were not significant. (c) RTs in milliseconds are displayed on the $Y$ axis as a function of congruence or incongruence of the current trial ( or $\mathrm{C}$ ), regardless of prior trial type. (d) RTs in milliseconds are displayed on the $Y$ axis as a function of trial repetition ( $\mathrm{cC}$ or il) or trial alternation ( $\mathrm{cl}$ or iC).

probability of detecting the observed group effects $(\beta \neq 0)$ given the data. This probability is displayed in a Posterior Probability Map. Because the Bayesian method infers posterior probability, by definition, it does not generate false positives and therefore does not require adjustment of its $p$-values based on $p$-value thresholding, as shown previously (Friston and Penny, 2003; Marsh et al, 2011). We adopted a similar approach as in Klein et al (2007) but with a more stringent threshold.

Because Bayesian-inference methods do not require corrections for multiple comparisons, they do not need to account for spatial features to derive statistical significance as in classical parametric-inference methods, which typically need corrections that assume a Gaussian random field or that take into account the cluster extent. As an additional requisite for assuming statistical significance and meaningful findings, however, we required positive findings to have a posterior probability greater than $97.5 \%$ and an extent of at least eight contiguous voxels in all our analyses (making our requisite for significance more stringent and discarding results comprising few voxels that, while statistically valid, have questionable biological significance).

We chose a Bayesian approach because it allows us to test directly our hypotheses, rather than to simply reject the null hypothesis (Stephens and Balding, 2009; Friston et al, 2002; Herzog and Ostwald, 2013; Woolrich et al, 2009). Second, this method is very reliable in comparing different groups of participants insofar as individual contributions to group-level estimates are weighted by the variability found within each participant. Third, we regard the Bayesian approach to be highly appropriate for detecting effects in cohorts that are by definition heterogeneous, as is the case in CHR cohorts, where the risk in statistical analyses of missing detection of true biological abnormalities (type II error) may be as important as reporting false positive findings (type I error).

Additionally, we cross-validated the results generated with Bayesian approach by using a brain-wide frequentist approach. Specifically, we performed a two-sample $t$-test of patients $v s$ controls in SPM8 and applied cluster thresholding based on Monte Carlo simulations as implemented previously (Slotnick et al, 2003) to correct for multiple comparisons. Our main results survived in the DLPFC with a height threshold of 0.004 in combination with a cluster threshold of 22 (yielding a corrected $p$-value of 0.05 ).

Finally, to quantify the accurateness and robustness of our estimates we conducted a leave-one-out jackknife resampling for unbiased estimation of effects (Abdi and Williams, 2010). We used this resampling technique to estimate the group effect (CHR individuals $v s$ Controls) for the main contrast $(\mathrm{cI}-\mathrm{cC})$, our main a priori test.

\section{Clinical, Behavioral, and Functional Associations}

Similar to others (Marsh et al, 2011; Klein et al, 2007), we did not use a Bayesian approach for clinical and behavioral associations and for associations with functional outcome, 


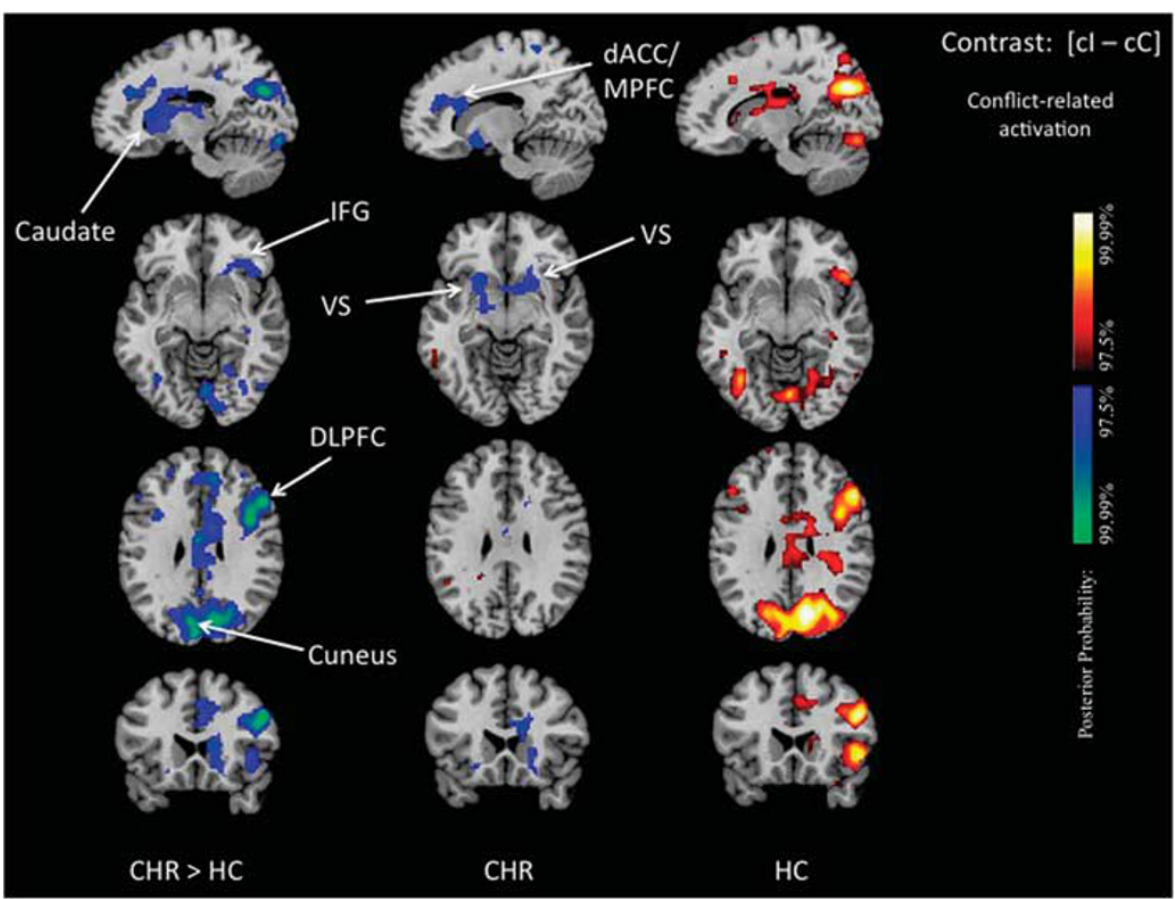

Figure 2 Group differences in conflict-related activation. We show posterior probability maps, which we thresholded at $97.5 \%$. Increases in signal during correct responses to $\mathrm{cl}$ relative to $\mathrm{CC}$ trials are shown in red and decreases in blue. This group comparison shows decreased conflict-related activations in $\mathrm{CHR}$ participants compared with healthy controls in the dorsal caudate, IFG, ventral striatum, the DLPFC, the dACC/MPFC and at the border between cuneus and precuneus (left column). Within-group effects are displayed separately for CHR patient (middle column) and healthy controls (right column) and confirm that the reduced conflict-related activations detected in the between-group comparison were driven by positive activations in controls and, in CHR participants, either by significant deactivations in the dACC and ventral striatum or, for the other regions, by a lack of detectable conflict-related activations. The reduced conflictrelated activations found in the CHR group represented areas where functional activity associated with the conflict-free condition (cC) was higher than that associated with the conflict-dependent condition (cl). dACC/MPFC, dorsal anterior cingulate/medial prefrontal cortex; DLPFC, dorsolateral prefrontal cortex; IFG, inferior frontal gyrus; VS, ventral striatum.

because such methods have only been developed and validated for the equivalent of $t$-tests but not for correlations. For brain-behavior associations, we correlated interference scores with the beta values from the conflict-related contrast. We applied cluster-extent thresholding based on Monte Carlo simulations (Slotnick et al, 2003) to correct $p$-values for multiple comparisons. We reach a corrected $p$-value (corrected for multiple comparisons) of $p<0.05$ by using the conjoint requirement of $p<0.025$ and a cluster extent threshold of at least 30 voxels (Forman et al, 1995). We further confirmed that our results did not change by also applying a smaller $p$-value (0.0125) (still yielding an effective $p$-value of <0.05). Correlations with the severity scores for clinical symptoms were performed in a similar manner. We used severity scores for the five items on the SIPS capturing positive symptoms as well as the total score for positive symptoms, given that both identification of CHR participants and conversion to psychosis are based on scores for positive symptoms. We applied Bonferroni correction for the six items tested. We conducted a comparison of conflictrelated activations between CHR individuals who developed psychosis (11 converters) and those who did not develop psychosis (45 non-converters). We also correlated conflictrelated activations with measures of social and role outcome (GSF:S and GSF:R, respectively). Finally, we checked for influential outliers by using a Cook's distance $D_{\mathrm{i}}$ criterion of $D_{\mathrm{i}}>1$. No influential outliers were found in the behavioral or imaging data.

\section{RESULTS}

\section{Behavioral Results}

No significant effect of group or of the interaction between group and trial type was detected on RTs or accuracy (all $p>0.57$ ), indicating the lack of difference between groups in performance (Figure 1). There was, however, an interaction between current trial type and prior trial type on RTs $(p<0.0001)$, demonstrating conflict adaptation effects, whereby the effects of current congruence on RTs were modulated by congruence on the previous trial, consistent with previous observations (Egner, 2007). Mean RTs increased in a curvilinear manner from the least demanding (cC) to the most demanding of the four trial types (cI). Repetitions of trial type (either $\mathrm{cC}$ or iI) were associated with shorter RTs than were trial alternations (cI or iC) $(p<0.0001)$. No significant interaction of trial repetition or alternation with group membership was observed. Interference scores did not differ significantly between CHR participants and healthy controls $(p=0.394)$, showing that CHR participants and controls were closely matched behaviorally for all effects. Finally, we found no significant difference in RTs or accuracy between CHR converters and CHR non-converters using parametric or non-parametric tests, demonstrating that both CHR subgroups were behaviorally matched (all $p>0.52$ ). 


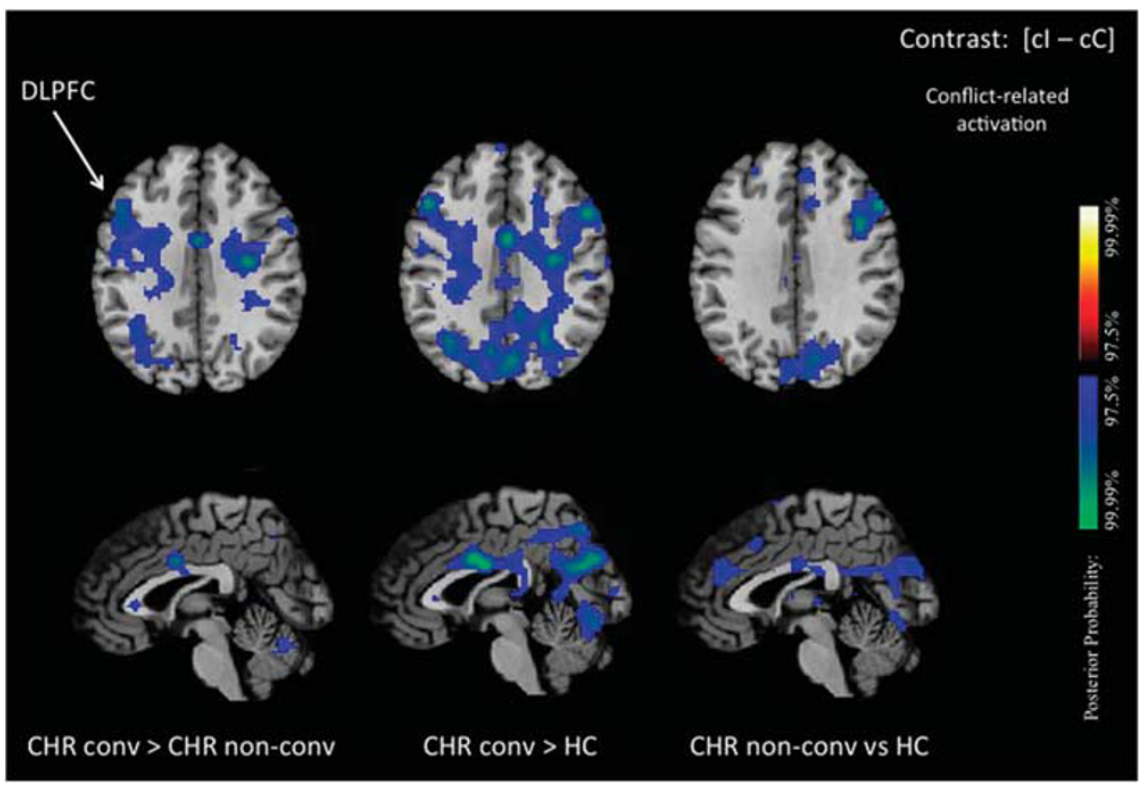

Figure 3 Conflict-related activation in converters. We show posterior probability maps, which we thresholded at $97.5 \%$. Increases in signal during correct responses to $\mathrm{cl}$ relative to $\mathrm{CC}$ trials are shown in red and decreases in blue. This group comparison between $\mathrm{CHR}$ participants who developed psychosis (converters) and CHR participants who did not develop psychosis (non-converters) shows reduced conflict-related activations in converters compared with non-converters in the DLPFC and midcingulate cortex (left column). Between-group comparisons with healthy controls are displayed separately for converters (middle column) and for non-converters (right column). This figure confirms that the reduction in activation difference between conflict-dependent and conflictfree trial that we observe in CHR participants is more pronounced in the DLPFC of CHR participants that ultimately develop psychosis. DLPFC, dorsolateral prefrontal cortex; MCC, midcingulate cortex.

\section{fMRI Results}

Conflict-related activation. Our main a priori contrast revealed reduced conflict-related activations in CHR participants, when compared with controls, in the head of the right caudate nucleus, the ventral striatum (VS), dACC, DLPFC, inferior frontal gyrus (IFG), and between cuneus and precuneus. Group differences in DLPFC and VS effect peaks had a moderate effect size (MNI coordinates $[x, y, z]$ in $\mathrm{mm}: 51,20,34$, and $-15,13,-8 ; Z$ scores: -3.94 and -2.19 jackknife estimates of $Z$ scores: -5.940 and -3.057 ; $95 \%$ confidence intervals: $[-3.5984,-8.2834]$ and $[-1.0202$, - 5.0947]; Cohen's $d$ : 0.7686 and 0.3976, respectively). Within-group analyses revealed that these between-group differences were driven by significant deactivations in the dACC and VS and, for the other regions, by the absence of significant conflict-related activations in the CHR participants that were present in controls. Plots of parameter estimates for each of these regions confirmed either the reduced or unchanged neural activation in the conflictdependent trial (cI) compared with the conflict-free trial (cC) in CHR participants. Controls, in contrast, showed greater neural activity in $\mathrm{cI}$ compared with $\mathrm{cC}$ trials, which produced positive conflict-related activations (Figure 2, Supplementary Figures S2 and S3).

Neural correlates of inter-individual differences in behavioral interference. In the control group, interference scores correlated inversely with conflict-related activation. The lower the interference score (ie, the better the performance on the task), the greater the conflict-related activation in DLPFC, dACC, thalamus, dorsal (but not ventral) striatum, and parietal cortex, indicating that more conflict-related activation in these regions was associated with less interference, or with better performance when processing cognitive conflict. This correlation was not detectable in the CHR group (Supplementary Figure S4).

\section{Correlations of Conflict-related Activity with Symptoms}

We did not observe any significant association with clinical symptoms.

\section{Association with Longitudinal Outcome}

Conversion. When we compared converters with nonconverters, converters exhibited reduced conflict-related activation in the DLPFC compared with non-converters (Figure 3).

Functional outcome. In the CHR group, higher levels of conflict-related activation in the DLPFC and in the fusiform gyrus were associated with better social outcome. Greater levels of conflict-related activation in the superior parietal cortex, the fusiform gyrus, the AACC, and the PCC correlated with better role outcome.

\section{DISCUSSION}

The aim of this study was to investigate the neural processing underlying cognitive control, specifically the processing of cognitive conflict, in participants at CHR for psychosis. We found reduced conflict-related activations in CHR individuals compared with healthy controls in the ventral and dorsal striatum, and in the DLPFC, IFG, dACC, and cuneus. 
Given that smaller conflict-related activations were associated longitudinally with conversion to psychosis and worse functional outcome, we interpreted these findings as evidence that conflict-related brain activation represents an adaptive process that is diminished in CHR individuals.

Post hoc analyses suggested that the reduced activation in the CHR group derived from activation during the conflictfree (congruent) trials that were equal or even greater than the activation associated with conflict-dependent (incongruent) trials (see Supplementary Figures S2 and S3). These findings suggest that CHR individuals generate a similar level of non-specific functional activity during any task condition, regardless of the presence of conflict, which in turn reduces the difference or contrast between conflict-dependent and conflict-free processing that we measure as reduced conflictdependent activation. We cannot determine definitively from our data set, however, whether $\mathrm{CHR}$ individuals fail to increase cortical activity in response to increased conflict or whether they simply respond to conflict-free trial as if they were conflict-laden trials (Supplementary Figure S2). We suspect, however, that conflict-free activation may have been higher in CHR individuals than in controls, because parameter estimates during the conflict-free condition were, at a trend level, greater in CHR individuals (refer to Supplementary Material) and were not significantly different for the conflict-dependent trials. Despite the challenges in interpreting activation during a single condition in the absence of an appropriate control condition, we speculate that these results could potentially reflect misallocation of cognitive resources toward less relevant or less demanding conditions (conflict-free trial in this case), in line with salience misattribution models of psychosis (Kapur, 2003).

The cortical regions of CHR individuals, where conflictrelated activations were abnormal (such as the DLPFC), were those that related to conversion to psychosis and functional outcome. These regions belong to frontostriatal networks found repeatedly to be abnormal in PET studies of CHR cohorts (Fusar-Poli et al, 2012). Our finding that the CHR and control groups did not differ in terms of behavioral performance is neither surprising (Wilkinson and Halligan, 2004), as our task had low cognitive demand by design, nor unique to our at-risk cohort (Yaakub et al, 2013).

We also observed reduced conflict-related activation in the VS. Though it is true that the VS has been thought to be mostly associated with reward, recent evidence suggests that this region has a broader role in information processing. Studies have shown, for instance, that conflict is processed as an aversive event, similar to a form of punishment (van Steenbergen et al, 2009), which we would expect to engage the reward system. These studies have found that while reward between trials abolishes adaptation effects, neutral or punishing events do not. Finally, some evidence points to a role of dopamine in cognitive control, explaining in part the patterns of brain activation we have seen (Braver et al, 1999; Montague et al, 2004; van Schouwenburg et al, 2010; Holroyd and Coles, 2002).

Our task was designed to have sufficiently low cognitive demand to ensure that the subjects were behaviorally matched despite the presence of prodromal symptoms that can impair performance on cognitive tasks, yet sufficiently demanding to generate detectable conflict signals in the brain. Behavioral matching allows us to unconfound the effects of different levels of task performance. If CHR subjects differed behaviorally, then it would be impossible to disentangle group effects from performance effects. Thus, when interpreting brain activation maps, we would not be able to say whether the brain activation differences are due solely to differences in performance, as healthy poor performers would typically show neural differences compared with healthy good performers, or to diagnosis, which would be perfectly confounded. Because participants and controls were matched on performance, we were able to attribute group effects to primary differences in neural processing that supports conflict processing, rather than to differences that are a consequence of poor performance. $\mathrm{CHR}$ converters were also performance-matched with $\mathrm{CHR}$ non-converters, thereby facilitating a clearer interpretation of the imaging findings specific to those individuals who developed psychotic illness. In a way, our approach has a similar rationale to that underlying the use of an exercise stress test in medicine as a cardiovascular challenge to find dormant heart disease (where the behavior on the task is running on a treadmill and the physiological readout is ECG abnormalities after reaching a target heart rate). In this case, the main point of the test is not to find abnormal behavior (that the person has trouble running or runs more slowly) but to challenge heart function to the point where abnormalities affecting its function are uncovered, despite apparent normal behavior.

The brain regions isolated by the conflict-related contrast are approximately the same regions in which conflict-related functional activation correlated with behavioral performance (Supplementary Figure S4). One notable exception was the $\mathrm{VS}$, though this was not surprising, given that this region is predominantly involved in reward processing, and we would not expect a clear association with the level of cognitive performance. The reward processing functions of the VS do not exclude its general involvement in the neural processing of conflict (Figure 2), however, as conflict itself can be experienced as a punishment. (van Steenbergen et al, 2009; Shenhav et al, 2013). We demonstrated that the greater the conflict-related activations in healthy participants, the better was their behavioral performance, consistent with the known activation of these regions in conflict situations (Carter et al, 2001). We were unable to detect a significant association between functional activation and performance in $\mathrm{CHR}$ participants.

The direct comparison of conflict-related activations in converters and non-converters suggests that reduced activation in the DLPFC when processing conflict is related to the development of psychosis, consistent with anatomical abnormalities in this region that have been associated with psychosis (Sun et al, 2009). The development of psychotic illness may therefore be related to an imaging phenotype that is characterized by reduced conflict-related activations in the DLPFC. Consistent with this interpretation, better longitudinal outcome in this cohort was associated with greater conflict-related activation in the DLPFC, ACC, PCC, and parietal cortex.

Our conversion rate $(20.7 \%)$, was similar to that in samples similar to ours in size (Yaakub et al, 2013) but larger than in some other published studies (Dandash et al, 2013; McFarlane et al, 2015). This rate is now considered as typical of prodromal research in general (Niendam et al, 2013; 
Yaakub et al, 2013; Katsura et al, 2014) and similar to the conversion rates in various consortia, such as the European Prediction of Psychosis Study (Salokangas et al, 2012). These rates reflect a decline in conversion since the time of the original reports in CHR samples (Yung et al, 2007). Our rate of exposure to antipsychotic medications and stimulant medications is also similar to other cohorts. We did not observe any significant association of clinical symptoms with the conflict-related contrast, likely because the correlations were too weak to survive correction for multiple comparisons. Our findings did not differ when we excluded participants taking antipsychotic medications and participants taking stimulants (Supplementary Figure S6), leading us to believe that medications were not a significant confounder.

Several limitations of our study should be noted. First, the absolute number of CHR subjects and converters may contribute to Type II error (the failure to detect real differences), even though our sample is fairly large for an imaging study and our conversion rate was similar to other cohorts. Also contributing to possible Type II error is the likelihood, if not certainty, that effect sizes will be smaller in at-risk cohorts than in a sample of individuals who already have an established psychotic illness. This is likely why we were unable to detect significant correlations between conflict-related activity and positive symptoms after correcting for multiple comparisons. Second, we cannot exclude entirely the possibility that medications may have influenced our findings, although our results did not change meaningfully when we excluded those participants who were taking antipsychotic or stimulant medications.

In conclusion, our study suggests that psychosis risk is associated at the neural level with reduced conflict-related brain activity. This neural phenotype appears to correlate within the DLPFC with the development of psychosis and with functional outcome.

\section{FUNDING AND DISCLOSURE}

Dr. Girgis has received research support from Otsuka and Genentech. All other authors declare no conflict of interest.

\section{ACKNOWLEDGMENTS}

This work was supported by NIMH grants 5T32MH15144, and K23 MH85063, K23 MH101637, UL1 RR024156, R21MH086125 and by funding from the National Alliance for Research on Schizophrenia and Depression (NARSAD), the Sackler Institute, the Herbert Irving Scholar Award, and the Bodini Fellowship at Columbia University. Dr Girgis has received research support from Ostuka and Genentech. The funders had no role in the design and conduct of the study, the collection, management, analysis, and interpretation of the data, the preparation, review, or approval of the manuscript and the decision to submit the manuscript for publication.

\section{REFERENCES}

Abdi H, Williams LJ (2010). Jackknife. In: Salkind NJ, Dougherty DM, Frey B., (eds). Encyclopedia of Research Design. Sage: Thousand Oaks, CA, pp 655-660.
Allen P, Luigjes J, Howes OD, Egerton A, Hirao K, Valli I et al (2012). Transition to psychosis associated with prefrontal and subcortical dysfunction in ultra high-risk individuals. Schizophr Bull 38: 1268-1276.

Botvinick M, Nystrom LE, Fissell K, Carter CS, Cohen JD (1999). Conflict monitoring versus selection-for-action in anterior cingulate cortex. Nature 402: 179-181.

Braver TS, Barch DM, Cohen JD (1999). Cognition and control in schizophrenia: a computational model of dopamine and prefrontal function. Biol Psychiatry 46: 312-328.

Cannon TD, Cadenhead K, Cornblatt B, Woods SW, Addington J, Walker E et al (2008). Prediction of psychosis in youth at high clinical risk: a multisite longitudinal study in North America. Arch Gen Psychiatry 65: 28-37.

Carrion RE, Goldberg TE, McLaughlin D, Auther AM, Correll CU, Cornblatt BA (2011). Impact of neurocognition on social and role functioning in individuals at clinical high risk for psychosis. Am J Psychiatry 168: 806-813.

Carrion RE, McLaughlin D, Goldberg TE, Auther AM, Olsen RH, Olvet DM et al (2013). Prediction of functional outcome in individuals at clinical high risk for psychosis. JAMA Psychiatry 70: 1133-1142.

Carter CS, MacDonald AW 3rd, Ross LL, Stenger VA (2001). Anterior cingulate cortex activity and impaired self-monitoring of performance in patients with schizophrenia: an event-related fMRI study. Am J Psychiatry 158: 1423-1428.

Cornblatt BA, Auther AM, Niendam T, Smith CW, Zinberg J, Bearden CE et al (2007). Preliminary findings for two new measures of social and role functioning in the prodromal phase of schizophrenia. Schizophr Bull 33: 688-702.

Crone EA, Dahl RE (2012). Understanding adolescence as a period of social-affective engagement and goal flexibility. Nat Rev Neurosci 13: 636-650.

Dandash O, Fornito A, Lee J, Keefe RS, Chee MW, Adcock RA et al (2013). Altered striatal functional connectivity in subjects with an at-risk mental state for psychosis. Schizophr Bull 40: 904-913.

Egner T (2007). Congruency sequence effects and cognitive control. CognAffect Behav Neurosci 7: 380-390.

Egner T (2008). Multiple conflict-driven control mechanisms in the human brain. Trends Cogn Sci 12: 374-380.

Forman SD, Cohen JD, Fitzgerald M, Eddy WF, Mintun MA, Noll DC (1995). Improved assessment of significant activation in functional magnetic resonance imaging (fMRI): use of a clustersize threshold. Magne Reson Med 33: 636-647.

Fornito A, Harrison BJ, Goodby E, Dean A, Ooi C, Nathan PJ et al (2013). Functional dysconnectivity of corticostriatal circuitry as a risk phenotype for psychosis. JAMA Psychiatry 70: 1143-1151.

Friston KJ, Glaser DE, Henson RN, Kiebel S, Phillips C, Ashburner J (2002). Classical and Bayesian inference in neuroimaging: applications. NeuroImage 16: 484-512.

Friston KJ, Penny W (2003). Posterior probability maps and SPMs. NeuroImage 19: 1240-1249.

Fusar-Poli P, Howes OD, Allen P, Broome M, Valli I, Asselin MC et al (2010). Abnormal frontostriatal interactions in people with prodromal signs of psychosis: a multimodal imaging study. Archives Gen Psychiatry 67: 683-691.

Fusar-Poli P, Howes OD, Allen P, Broome M, Valli I, Asselin MC et al (2011). Abnormal prefrontal activation directly related to pre-synaptic striatal dopamine dysfunction in people at clinical high risk for psychosis. Mol Psychiatry 16: 67-75.

Fusar-Poli P, McGuire P, Borgwardt S (2012). Mapping prodromal psychosis: a critical review of neuroimaging studies. Eur Psychiatry 27: 181-191.

Green MF (1996). What are the functional consequences of neurocognitive deficits in schizophrenia? Am J Psychiatry 153: 321-330.

Herzog S, Ostwald D (2013). Experimental biology: sometimes Bayesian statistics are better. Nature 494: 35. 
Holroyd CB, Coles MG (2002). The neural basis of human error processing: reinforcement learning, dopamine, and the errorrelated negativity. Psychol Rev 109: 679-709.

Horga G, Maia TV, Wang P, Wang Z, Marsh R, Peterson BS (2011). Adaptation to conflict via context-driven anticipatory signals in the dorsomedial prefrontal cortex. J Neurosci 31: 16208-16216.

Kapur S (2003). Psychosis as a state of aberrant salience: a framework linking biology, phenomenology, and pharmacology in schizophrenia. Am J Psychiatry 160: 13-23.

Katsura M, Ohmuro N, Obara C, Kikuchi T, Ito F, Miyakoshi T et al (2014). A naturalistic longitudinal study of at-risk mental state with a 2.4 year follow-up at a specialized clinic setting in Japan. Schizophr Res 158: 32-38.

Kerns JG (2006). Anterior cingulate and prefrontal cortex activity in an FMRI study of trial-to-trial adjustments on the Simon task. NeuroImage. 33: 399-405.

Kerns JG, Cohen JD, MacDonald AW 3rd, Cho RY, Stenger VA, Carter CS (2004). Anterior cingulate conflict monitoring and adjustments in control. Science 303: 1023-1026.

Klein TA, Neumann J, Reuter M, Hennig J, von Cramon DY, Ullsperger M (2007). Genetically determined differences in learning from errors. Science 318: 1642-1645.

Lesh TA, Niendam TA, Minzenberg MJ, Carter CS (2011). Cognitive control deficits in schizophrenia: mechanisms and meaning. Neuropsychopharmacology 36: 316-338.

Luna B (2009). Developmental changes in cognitive control through adolescence. Adv Child Dev Behav 37: 233-278.

Marsh R, Horga G, Parashar N, Wang Z, Peterson BS, Simpson HB (2013). Altered activation in fronto-striatal circuits during sequential processing of conflict in unmedicated adults with obsessive-compulsive disorder. Biol Psychiatry 75: 615-622.

Marsh R, Horga G, Wang Z, Wang P, Klahr KW, Berner LA et al (2011). An FMRI study of self-regulatory control and conflict resolution in adolescents with bulimia nervosa. Am J Psychiatry 168: $1210-1220$.

Marsh R, Maia TV, Peterson BS (2009). Functional disturbances within frontostriatal circuits across multiple childhood psychopathologies. Am J Psychiatry 166: 664-674.

Matthews M, Bondi C, Torres G, Moghaddam B (2013). Reduced presynaptic dopamine activity in adolescent dorsal striatum. Neuropsychopharmacology 38: 1344-1351.

McFarlane WR, Levin B, Travis L, Lucas FL, Lynch S, Verdi M et al (2015). Clinical and functional outcomes after 2 years in the early detection and intervention for the prevention of psychosis multisite effectiveness trial. Schizophr Bull 41: 30-43.

Miller TJ, McGlashan TH, Rosen JL, Cadenhead K, Cannon T, Ventura J et al (2003). Prodromal assessment with the structured interview for prodromal syndromes and the scale of prodromal symptoms: predictive validity, interrater reliability, and training to reliability. Schizophr Bull 29: 703-715.

Montague PR, Hyman SE, Cohen JD (2004). Computational roles for dopamine in behavioural control. Nature 431: 760-767.

Naneix F, Marchand AR, Pichon A, Pape JR, Coutureau E (2013). Adolescent stimulation of D2 receptors alters the maturation of dopamine-dependent goal-directed behavior. Neuropsychopharmacology 38: 1566-1574.
Neumann J, Lohmann G (2003). Bayesian second-level analysis of functional magnetic resonance images. NeuroImage 20: $1346-1355$.

Niendam TA, Lesh TA, Yoon J, Westphal AJ, Hutchison N, Daniel R J et al (2013). Impaired context processing as a potential marker of psychosis risk state. Psychiatry Res 221: 13-20.

Paus T, Keshavan M, Giedd JN (2008). Why do many psychiatric disorders emerge during adolescence? Nat Rev Neurosci 9: 947-957.

Rubia K, Smith AB, Woolley J, Nosarti C, Heyman I, Taylor E et al (2006). Progressive increase of frontostriatal brain activation from childhood to adulthood during event-related tasks of cognitive control. Hum Brain Mapp 27: 973-993.

Salokangas RK, Ruhrmann S, von Reventlow HG, Heinimaa M, Svirskis T, From T et al (2012). Axis I diagnoses and transition to psychosis in clinical high-risk patients EPOS project: prospective follow-up of 245 clinical high-risk outpatients in four countries. Schizophr Res 138: 192-197.

Shenhav A, Botvinick MM, Cohen JD (2013). The expected value of control: an integrative theory of anterior cingulate cortex function. Neuron 79: 217-240.

Slotnick SD, Moo LR, Segal JB, Hart J Jr. (2003). Distinct prefrontal cortex activity associated with item memory and source memory for visual shapes. Brain Res Cogn Brain Res 17: 75-82.

Snitz BE, Macdonald AW 3rd, Carter CS (2006). Cognitive deficits in unaffected first-degree relatives of schizophrenia patients: a meta-analytic review of putative endophenotypes. Schizophr Bull 32: 179-194.

Stephens M, Balding DJ (2009). Bayesian statistical methods for genetic association studies. Nat Rev Genet 10: 681-690.

Sun D, Phillips L, Velakoulis D, Yung A, McGorry PD, Wood SJ et al (2009). Progressive brain structural changes mapped as psychosis develops in 'at risk' individuals. Schizophr Res 108: 85-92.

van Schouwenburg M, Aarts E, Cools R (2010). Dopaminergic modulation of cognitive control: distinct roles for the prefrontal cortex and the basal ganglia. Curr Pharm Des 16: 2026-2032.

van Steenbergen H, Band GP, Hommel B (2009). Reward counteracts conflict adaptation. Evidence for a role of affect in executive control. Psychol Sci 20: 1473-1477.

Wilkinson D, Halligan P (2004). The relevance of behavioural measures for functional-imaging studies of cognition. Nat Rev Neurosci 5: 67-73.

Wood SJ, Pantelis C, Proffitt T, Phillips LJ, Stuart GW, Buchanan JA et al (2003). Spatial working memory ability is a marker of risk-forpsychosis. Psychol Med 33: 1239-1247.

Woolrich MW, Jbabdi S, Patenaude B, Chappell M, Makni S, Behrens $\mathrm{T}$ et al (2009). Bayesian analysis of neuroimaging data in FSL. NeuroImage 45: S173-S186.

Yaakub SN, Dorairaj K, Poh JS, Asplund CL, Krishnan R, Lee J et al (2013). Preserved working memory and altered brain activation in persons at risk for psychosis. Am J Psychiatry 170: 1297-1307.

Yung AR, McGorry PD (1996). The prodromal phase of firstepisode psychosis: past and current conceptualizations. Schizophr Bull 22: 353-370.

Yung AR, Yuen HP, Berger G, Francey S, Hung TC, Nelson B et al (2007). Declining transition rate in ultra high risk (prodromal) services: dilution or reduction of risk? Schizophr Bull 33: 673-681.

Supplementary Information accompanies the paper on the Neuropsychopharmacology website (http://www.nature.com/npp) 